Confidentiality and a 'no blame' ethos are key principles when reviewing cases where shortcomings are identified. The information obtained during each $\mathrm{M}+\mathrm{M}$ meeting is then stored in its PowerPoint format in a confidential file held by our Administrative Head of Department.

Data collected for each of the meetings was reviewed by the authors and collated

Results The number of cases discussed at each meeting ranged from 24- 42 with a mean of 30 . Interestingly there was no seasonal variation in total numbers of transfers or deaths. A wide variety of pathologies were seen. The number of deaths over each three month period remained markedly consistent both in terms of numbers at four and causes- expected deaths due to life limiting illness, accidents, suicide, sepsis and SIDS . Conclusion We established a Paediatric $\mathrm{M}+\mathrm{M}$ meeting to review complicated cases and deaths at our busy Regional Centre. Our study suggests that findings in relation to Paediatric serious illness and death have remained consistent in our catchment area since our successful initiative with no obvious trends.

\section{P173 ACT BEST ACTIVE CLINICAL TRIAGING BEFORE EVALUATION EXPEDITES TREATMENT}

${ }^{1,2}$ Jean Donnelly, ${ }^{1,3}$ Suzanne Kelleher*. 'Our Lady's Children's Hospital Crumlin, Dublin, Ireland; ${ }^{2}$ Trinity College Dublin, Dublin, Ireland; ${ }^{3}$ Royal College of Surgeons Ireland, Dublin, Ireland

\subsection{6/archdischild-2019-epa.528}

Aim To improve patient safety while on an OPD waiting list, by implementing an active triaging process

Method The traditional triage process for referrals was passive, subjective and varied amongst consultants resulting in the majority of children being waitlisted. All new referrals to General Paediatrics were reviewed in a Triage Assessment clinic by two consultants, who jointly reviewed each referral in conjunction with any additional patient information available e.g. previous attendances, investigations etc. They were then waitlisted or deflected back to the GP or to other more appropriate services. Patient 'activities' were performed as appropriate, including radiology, phlebotomy or providing treatment algorithms to $\mathrm{GP} /$ parents. A record was kept of all investigations.

Results All data was collected for analysis including number of referrals, triage outcome, reason for referral, activities performed. Analysis of data allowed us to determine the change in triage outcomes. During the initial 16 months of the project 1900 referrals were assessed. Of these, 1203 (63\%) were waitlisted, 580 (31\%) were deflected/rejected and $114(6 \%)$ were 'Out of Catchment'. Of those waitlisted 830 (69\%) were routine, $313(26 \%)$ were soon and 59 (5\%) were urgent. Overall, 1109 activities were recorded including sending letters to GPs (588) and parents (105), ordering radiologic investigations (135), phoning GPs (46) or parents (119) for further information. The project has decreased by $37 \%$ those waitlisted for OPD appointments.

Conclusion The waiting list should decrease the waiting list by 13.6 months year on year as 694 less new patient appointments were needed over the first 16 months.

The impact on patients was that six serious illnesses were detected early who otherwise would have been delayed in their diagnoses and management with serious consequences.

Patient care from the first point of contact with the hospital is now vastly improved from a patient safety perspective.
GPs have welcomed direct responsive engagement with hospital consultants. This project is projected to be rolled out nationally. Expanding this project beyond our own department will prove challenging as it's a new way of working.

\section{P174 A STUDY OF END OF LIFE CARE ALGORITHMS FOLLOWING THE DEATH OF A CHILD IN A TERTIARY PAEDIATRIC HOSPITAL}

${ }^{1}$ Amy P Worrall*, ${ }^{1}$ Eleanor Gallagher, ${ }^{2}$ Maeve O'Reilly, ${ }^{2}$ Marie Twomey, ${ }^{2}$ Mary Devins. ${ }^{1}$ School of Medicine, Trinity College Dublin, Dublin, Ireland; ' $O u r$ Lady's Children's Hospital Crumlin, Dublin, Ireland

\subsection{6/archdischild-2019-epa.529}

Aims Prompt communication with all professionals involved in the care of a child who has died is essential. To aid this, Our Lady's Children's Hospital Crumlin (OLCHC), has developed a pan departmental algorithm to provide guidance. The 'When a Child Dies (WACD) Algorithm' provides a guided checklist for staff to ensure all stakeholders are informed of the death, and that tasks related to the care of the child and family following a death are completed. This research aimed to audit the activation and completion of the WACD algorithms.

Methods A retrospective study was conducted in a 324-bed in OLCHC. The deaths of 251 children from 2015-2016 were included. Demographic information, diagnoses, disease status, end of life care, place of death and algorithm completion was investigated. The audit was conducted against standards from current institutional guidelines.

Results Of the 251 paediatric deaths audited, 43\% died in OLCHC, $11 \%$ died in another hospital or hospice, $3 \%$ were unaccounted for, and $42 \%$ died at home. The most common departments were Cardiology and Haematology/Oncology, accounting for $26.3 \%$ and $21.5 \%$ of deaths respectively. $17 \%$ (43) had the appropriate WACD fully completed, 48\% (120) were partially completed and $35 \%$ (88) of cases had no WACD forms completed.

Conclusions Departments that more commonly encounter paediatric deaths were more consistent in completion of the WACD algorithm. There is a need to disseminate information and knowledge of the algorithms, especially in departments with a low frequency of childhood deaths. This would ensure that primary care teams, GPs, referring hospitals and any other relevant healthcare professionals are informed on the death of their patient. It also ensures that families are met by the on-call team, the chaplain, and that follow-up with their primary consultant is arranged for the family.

\section{P175 REVIEW OF BREAKTHROUGH ANALGESIA PRESCRIBED USING TRANSDERMAL BUPENORPHINE AS THEIR BACKGROUND OPIOID, IN CHILDREN UNDER REVIEW BY PALLIATIVE MEDICINE IN AN INPATIENT SETTING}

Karen Dennehy*. St Luke's Hospital and Our Lady's Children's Hospital Crumlin, Dublin, Ireland

\subsection{6/archdischild-2019-epa.530}

Background Buprenorphine is a synthetic opioid analgesic, its primary therapeutic actions being analgesia and sedation. 